\title{
A Uniformly Convergent Numerical Method Using Weak Formulation for Singularly Perturbed Differential Equations
}

\author{
Weiqun Zhang \\ Lake Campus Science \& Mathematics, Wright State University, 7600 State Rt 703, Celina, OH 45822, USA
}

\begin{abstract}
A numerical method using weak formulation is proposed to solve singularly perturbed differential equations. The numerical method is applied to both linear and nonlinear perturbation problems. A linear differential equation is solved using its weak formulation with a test space composed of exponential functions matching boundary layers. A nonlinear singular perturbation problem is converted into a system of linear differentiation equations. Then each linear differential equation is solved iteratively. The uniform convergence, which is independent of the singular perturbation parameter, is numerically verified.
\end{abstract}

Key words: Singular perturbation, differential equations, boundary layers, numerical methods, weak formulation.

\section{Introduction}

We consider a singularly perturbed differential equation in its quasi-linear form:

$$
\begin{aligned}
& -\varepsilon u^{\prime \prime}+f(x, u) u^{\prime}=g(x, u) \text { for } x \in \Omega, \\
& u(a)=v_{a} \text { and } u(b)=v_{b},
\end{aligned}
$$

where $\varepsilon \ll 1$ is a positive singular perturbation parameter and $\Omega=(a, b)$. It is also assumed $f(x, u)$ and $g(x, u)$ are continuously differentiable and $f(x, u) \neq 0$. It is shown in Chang and Howes [1] the solution of such a singular perturbation problem is bounded. The a'priori bounds are further developed and improved by Zhang [2]. Thus boundary layers are detected via the a'priori bounds. For all $x \in \Omega$, a solution of the quasilinear singular perturbation problem has an exponential layer at the left boundary if $f(x, u) \leq-k<0$, or at the right boundary if $f(x, u) \geq k>0$ for all $x \in \Omega$, where $k$ is a positive constant. Moreover, either of the boundary layer length is proportional to the singular perturbation parameter $\varepsilon$. Because of the presence of boundary

Corresponding author: Weiqun Zhang, Ph.D., research fields: numerical analysis and scientific programming. layers, it requires special treatment in solving singular perturbation problems numerically. In general, there are two types of numerical methods to accommodate the boundary layers. One method is based on a non uniform mesh with common finite difference schemes, Linss et al. [3], Miller et al. [4], and Lin et al. [5]. Specifically, the non uniform mesh is adapted to the singular perturbation parameter such that it is dense on the layer and sparse elsewhere. The other is based on a uniform mesh, with adapted finite differences, Choudhury [6], Ilicasu and Schultz [7], and Segal [8]. In this paper, we will explore to solve the singular perturbation problem with the latter approach. First, a quasi-linear singular perturbation problem is converted into a system of linear differential equations. Then each linear differential equation is solved using its weak formulation with the test space chosen as exponential functions matching boundary layers.

\section{The Conversion from Quasilinear to Linear}

When applied with finite differences, a linear differential equation will turn into a system of linear 
equations and a nonlinear differential equation will turn into a nonlinear system. A quasi-linear singular perturbation problem can be solved when turned into a nonlinear system. It can also be solved when a quasi-linear singular perturbation problem is converted into linear. For our consideration, we will convert a quasi-linear singular perturbation problem into a sequence of linear singular perturbation problems. Then each linear singular perturbation problem is solved numerically using the method proposed in the next section. The converged solution of the linear ones will be that of the quasilinear singular perturbation problem.

Let $\left\{u_{[I]} \mid I=0,1,2, \ldots\right\}$ be a sequence of functions such that $u_{[I]}(a)=v_{a}, u_{[I]}(b)=v_{b}$, and $u_{[0]}(x)$ be the initial guess and $u_{[I+1]}(x)$ satisfy

$$
\begin{aligned}
& \varepsilon u_{[I+1]}^{\prime \prime}=f\left(x, u_{[I]}\right) u_{[I+1]}^{\prime}+h_{[I]}(x) u_{[I+1]}+r_{[I]}(x), \\
& u_{[I+1]}(a)=v_{a} \text { and } u_{[I+1]}(b)=v_{b},
\end{aligned}
$$

where

$$
\begin{aligned}
& h_{[I]}(x)=f_{u}\left(x, u_{[I]}\right) u_{[I]}^{\prime}+g_{u}\left(x, u_{[I]}\right), \\
& r_{[I]}(x)=g\left(x, u_{[I]}\right)-h_{[I]}(x) u_{[I]}(x),
\end{aligned}
$$

Let $\boldsymbol{u}(\boldsymbol{x})$ be the solution of the singular perturbation (1) and $u_{[0]}(x)$ be the initial guess which is sufficiently close to $\boldsymbol{u}(\boldsymbol{x})$. Then the sequence

$$
\left\{u_{[I]}(x) \mid I=0,1,2, \ldots\right\} \text { converges to } u(x) \text {. And }
$$

it converges at the quadratic rate, i.e.,

$$
u_{[I+1]}(x)-u(x)=O\left(u_{[I]}(x)-u(x)\right)^{2} \quad[9] .
$$

Note that Eq. (2) represents a sequence of linear differential equations. In the next section, we turn our attention to the singular perturbation in its linear form.

$$
\begin{aligned}
& \varepsilon u^{\prime \prime}=f(x) u^{\prime}+g(x) \text { for } x \in \Omega \text { and } f(x) \neq 0, \\
& u(a)=v_{a} \text { and } u(b)=v_{b} .
\end{aligned}
$$

\section{The Construction of a Uniformly Convergent Numerical Method}

From now on, we arrange the linear singular perturbation problem (3) in a new form:

$$
-\varepsilon u^{\prime \prime}+f(x) u^{\prime}=g(x) \text { for } x \in \Omega,
$$

where $\varepsilon \ll 1$ is a positive singular perturbation parameter and $\Omega=(0,1)$. Without loss of generality, assume the linear singular perturbation problem has the homogeneous boundary conditions, i.e. $v_{0}=$ $v_{1}=0$ and $f(x) \geq k>0$, where $k$ is a positive constant. Combining the derivative terms in the problem, define the linear differential operator $L$

$$
L u=-\varepsilon u^{\prime \prime}+f u^{\prime} .
$$

So the singular perturbation problem is now in the form of

$$
L u=g
$$

Let $H_{0}^{1}(\Omega)$ be the Sobolev space. For any $r, s \in H_{0}^{1}(\Omega)$ and we use the $L^{2}$ scalar product

$$
(r, s)=\int_{\Omega} r s d x .
$$

Accordingly, the singular perturbation problem is transformed in term of weak formulation, which is to find $u \in H_{0}^{1}(\Omega)$ such that for all $v \in H_{0}^{1}(\Omega)$,

$$
b(u, v)=(g, v)
$$

where $a$ is the continuous bilinear form for all $u, v \in H_{0}^{1}(\Omega)$. By choosing $v=0$ on the boundary and integrating by parts, we get

$$
b(u, v)=\left(\varepsilon u^{\prime}, v^{\prime}\right)+\left(f u^{\prime}, v\right) .
$$

It can be shown that the bilinear form $b(u, v)$ is bounded and coercive. Hence, there is a unique solution for the singular perturbation problem in its weak formulation (5) [10].

The continuous singular perturbation problem (4) is now discretized using its weak formulation (5). The mesh is the uniform mesh $\Omega^{n}=\left\{x_{i}\right\}_{0}^{n}$ with $h=\frac{1}{n}$.

For the coefficient function $f$ of the first derivative term $u^{\prime}$, a piecewise constant approximation $\bar{f}$ on $\Omega^{n}$, is defined, for each $i, 1 \leq i \leq n$, 


$$
\bar{f}(x)=\bar{f}_{i}, \text { for all } x \in\left(x_{i-1}, x_{i}\right],
$$

where the constants $\bar{f}_{i}=\frac{f\left(x_{i-1}\right)+f\left(x_{i}\right)}{2}$. Later on, we will denote $\bar{g}$ an approximation to the right hand side $g(x)$ in the linear singular perturbation problem (4), where $\bar{g}$ will be defined in the same fashion.

The test space is defined to be the space of piecewise exponential functions spanned by $\left\{v_{i}\right\}_{1}^{n-1}$. Specifically, for $1 \leq i \leq n-1$, we define

$$
v_{i}(x)=\left\{\begin{array}{c}
\frac{1-e^{-\tau_{i}\left(x-x_{i-1}\right) / h}}{1-e^{-\tau_{i}}} \text { for } x_{i-1} \leq x \leq x_{i}, \\
1-\frac{1-e^{-\tau_{i+1}\left(x-x_{j}\right) / h}}{1-e^{-\tau_{i+1}}} \text { for } x_{i} \leq x \leq x_{i+1}, \\
0, \text { otherwise }
\end{array}\right.
$$

where $\tau_{i}=\frac{\bar{f}_{i} h}{\varepsilon}$.

Accordingly, the derivatives of above functions can be easily found,

$$
v_{i}^{\prime}(x)=\left\{\begin{array}{l}
\frac{\tau_{i}}{h} \frac{e^{-\tau_{i}\left(x-x_{i-1}\right) / h}}{1-e^{-\tau_{i}}} \text { for } x_{i-1} \leq x \leq x_{i}, \\
-\frac{\tau_{i+1}}{h} \frac{e^{-\frac{\tau_{i+1}\left(x-x_{i}\right)}{h}}}{1-e^{-\tau_{i+1}}} \text { for } x_{i} \leq x \leq x_{i+1}, \\
0, \text { otherwise }
\end{array}\right.
$$

For the purpose of integrals in the weak formulation, we also calculate the following limits:

$$
\lim _{x \rightarrow x_{i-1}^{-}} v_{i}^{\prime}(x)=0
$$

and

$$
\lim _{x \rightarrow x_{i-1}^{+}} v_{i}^{\prime}(x)=\frac{\tau_{i}}{1-e^{-\tau_{i}}} .
$$

Here we let

$$
B(\tau)=\left\{\begin{array}{c}
\frac{\tau}{e^{\tau}-1}, \text { for } \tau \neq 0, \\
1, \text { for } \tau=0 .
\end{array}\right.
$$

So the above right sided limit can be written as

$$
\lim _{x \rightarrow x_{i-1}^{+}} v_{i}^{\prime}(x)=\frac{1}{h} B\left(-\tau_{i}\right) .
$$

Similarly,

$$
\begin{gathered}
\lim _{x \rightarrow x_{i}^{-}} v_{i}^{\prime}(x)=\frac{1}{h} B\left(\tau_{i}\right), \\
\lim _{x \rightarrow x_{i}^{+}} v_{i}^{\prime}(x)=-\frac{1}{h} B\left(-\tau_{i+1}\right),
\end{gathered}
$$

$$
\begin{gathered}
\lim _{x \rightarrow x_{i+1}^{-}} v_{i}^{\prime}(x)=-\frac{1}{h} B\left(\tau_{i+1}\right), \text { and } \\
\lim _{x \rightarrow x_{i+1}^{+}} v_{i}^{\prime}(x)=0 .
\end{gathered}
$$

We also have, for all $k$ satisfying $|k-i|>1$,

$$
\lim _{x \rightarrow x_{k}^{-}} v_{i}^{\prime}(x)=\lim _{x \rightarrow x_{k}^{+}} v_{i}^{\prime}(x)=0 .
$$

The discrete bilinear form $b_{n}$, corresponding to the bilinear form $b$ in the weak formulation (5), is now defined by

$$
b_{n}(u, v)=\left(\varepsilon u^{\prime}, v^{\prime}\right)+\left(\bar{f} u^{\prime}, v\right)
$$

From now on, we use $u_{\varepsilon}$ to denote the solution of the singular perturbation problem in the discrete bilinear form. Integrating by parts, it is clear that, for all $i, 1 \leq i \leq n-1$, the bilinear forms are given by

$$
\begin{gathered}
b_{n}\left(u_{\varepsilon}, v_{i}\right)=\left(u_{\varepsilon}^{\prime}, \varepsilon v_{i}^{\prime}+\bar{f} v_{i}\right) \\
=\int_{x_{i-1}}^{x_{i+1}} u_{\varepsilon}^{\prime}(x)\left(\varepsilon v_{i}^{\prime}+\bar{f} v_{i}\right)(x) d x \\
=\left.\left(\varepsilon v_{i}^{\prime}+\bar{f} v_{i}\right) u_{\varepsilon}\right|_{x_{i-1}} ^{x_{i}}+\left.\left(\varepsilon v_{i}^{\prime}+\bar{f} v_{i}\right) u_{\varepsilon}\right|_{x_{i}} ^{x_{i+1}} \\
=-\varepsilon\left[\lim _{x \rightarrow x_{i}^{+}} v_{i}^{\prime}(x)\right] u_{\varepsilon}\left(x_{i-1}\right)+ \\
{\left[\left(\varepsilon \lim _{x \rightarrow x_{i}^{-}} v_{i}^{\prime}(x)+\bar{f}_{i}\right)-\left(\varepsilon \lim _{x \rightarrow x_{i}^{+}} v_{i}^{\prime}(x)+\bar{f}_{i+1}\right)\right] u_{\varepsilon}\left(x_{i}\right)} \\
+\varepsilon\left[\lim _{x \rightarrow x_{i+1}^{-}} v_{i}^{\prime}(x)\right] u_{\varepsilon}\left(x_{i+1}\right) \\
= \\
\frac{\varepsilon}{h}\left[-B\left(\tau_{i+1}\right) u_{\varepsilon}\left(x_{i+1}\right)+\left(B\left(\tau_{i+1}\right)+\left(B\left(\tau_{i+1}\right) u_{\varepsilon}\left(x_{i}\right)-\right.\right.\right. \\
\left.B\left(-\tau_{i}\right) u_{\varepsilon}\left(x_{i-1}\right)\right] .
\end{gathered}
$$

For the right hand side of the weak formulation of the singular perturbation problems is discretized as follows.

$$
\begin{gathered}
(g, v)=(\bar{g}, v) \\
=\int_{x_{i-1}}^{x_{i+1}} \bar{g}_{i}(x) v_{i}(x) d x .
\end{gathered}
$$

Note $\bar{g}_{i}(x)=\bar{g}_{i}$ is a constant, we have

$$
\begin{aligned}
& \int_{x_{i-1}}^{x_{i}} \bar{g}_{i}(x) v_{i}(x) d x=\frac{h \bar{g}_{i}}{\tau_{i}}\left(B\left(-\tau_{i}\right)-1\right), \\
& \int_{x_{i}}^{x_{i+1}} \bar{g}_{i}(x) v_{i}(x) d x=\frac{h \bar{g}_{i}}{\tau_{i+1}}\left(1-B\left(\tau_{i+1}\right)\right) .
\end{aligned}
$$

Finally, the linear singular perturbation problems in 
its weak formulation (5) are discretized into a system of linear equations.

$$
A u_{\varepsilon}=\frac{h^{2}}{\varepsilon} R
$$

where $A=\left(a_{i j}\right)$ is a tridiagonal matrix of order $n-1$, with the non-zero elements of $A$ satisfying

$$
\begin{gathered}
a_{i i}=-\left(a_{i, i-1}+a_{i, i+1}\right), a_{i, i-1}<0, a_{i, i+1}<0, \\
R=\left(r_{i}\right) \text { is a column vector and } \\
r_{i}=\frac{\bar{g}_{i-1}}{2 \tau_{i}}\left(B\left(-\tau_{i}\right)-1\right)+\frac{\bar{g}_{i}}{2 \tau_{i}}\left(B\left(-\tau_{i}\right)-1\right)+\frac{\bar{g}_{i}}{2 \tau_{i+1}}(1- \\
\left.B\left(-\tau_{i+1}\right)\right)+ \\
\frac{\bar{g}_{i+1}}{2 \tau_{i+1}}\left(1-B\left(-\tau_{i+1}\right)\right) .
\end{gathered}
$$

It is clear that $A$ is an M-matriax, thus $A$ is invertible. Hence the linear system has a unique solution. The above consideration was first introduced by El-Mistikawy and Werle [11].

In this paper, we extend the scheme to quasi-linear singular perturbation problems. As outlined in the previous sections, a quasi-linear singular perturbation problem is first linearized into a system of linear differential equations. Each linear differential equation is solved numerically using the proposed scheme. Also it is worth to mention the test vectors are constructed to match the exponential boundary layers. The uniform convergence is verified with a numerical example in the next section.

\section{A Numerical Example}

First, we apply the new method to solve the linear problem

$$
\begin{aligned}
& \varepsilon u^{\prime \prime}=u^{\prime} \text { for } x \in(0,1), \\
& u(0)=0 \text { and } u(1)=1,
\end{aligned}
$$

with the exact solution $u(x)=\frac{e^{\frac{x-1}{\varepsilon}}-e^{\frac{-1}{\varepsilon}}}{1-e^{\frac{-1}{\varepsilon}}}$, which is used for numerical verification purpose.

The numerical solution of the singular perturbation problem (6) is compared with that of other methods.
To demonstrate the stability of the new method, a uniform mesh with weak formulation in this paper, we listed the maximal errors with different values of the singular perturbation parameter (Table 1). As expected and shown from the table, the new method is convergent and stable.

Finally, we solve the nonlinear singular perturbation problem,

$$
\begin{aligned}
& \varepsilon u^{\prime \prime}+e^{u} u^{\prime}-\frac{\pi}{2} \sin \frac{\pi x}{2} e^{2 u}=0 \text { for } x \in(0,1), \\
& u(0)=0 \text { and } u(1)=0,
\end{aligned}
$$

with the asymptotic expansion

$$
u(x, \varepsilon)=-\ln \left[\left(1+\cos \frac{\pi x}{2}\right)\left(1-\frac{1}{2} e^{-\frac{x}{2 \varepsilon}}\right)\right]+O(\varepsilon),
$$

[12], which is used numerical verification purpose only.

We expect a left boundary layer since $f(x, u)=-e^{u}<0$.

The linearized systems corresponding to the singular perturbation problem (7) are

$$
\begin{aligned}
& \varepsilon u_{[I+1]}^{\prime \prime}+a_{[I]}(x) u_{[I+1]}^{\prime}+b_{[I]}(x) u_{[I+1]} \\
& =f_{[I]}(x) \text { for } x \in(0,1)
\end{aligned}
$$

where

$$
\begin{aligned}
& a_{[I]}(x)=e^{u_{[I]}(x)}, \\
& b_{[I]}(x)=e^{u_{[I]}(x)} u_{[I]}^{\prime}(x)-\pi e^{2 u_{[I]}(x)} \sin \frac{\pi x}{2} \text { and } \\
& f_{[I]}(x)=\frac{\pi}{2} e^{2 u_{[I]}} \sin \frac{\pi x}{2}+b_{[I]}(x) u_{[I]}(x) .
\end{aligned}
$$

For each of the linear singular perturbation problems, we use its discretized weak formulation with the same test functions. The linear system of differential equations converges within dozens of iterations.

The numerical solution of the singular perturbation problem (7) is compared with that of other methods. To demonstrate the stability of the new method, a uniform mesh with weak formulation in this paper, we 
Table 1 Maximal error comparison among different methods.

\begin{tabular}{llllll}
\hline \multirow{2}{*}{ Method } & \multicolumn{5}{c}{ Maximal error } \\
\cline { 2 - 6 } & $\varepsilon=10^{-1}$ & $\varepsilon=10^{-2}$ & $\varepsilon=10^{-4}$ & $\varepsilon=10^{-8}$ & $\varepsilon=10^{-10}$ \\
\hline Choo and Schultz [13], 4th order & $1.13 * 10^{-6}$ & $7.72 * 10^{-5}$ & N.A. & N.A. & N.A. \\
Lin et al. [5], the central differences & $6.70 * 10^{-5}$ & $5.66 * 10^{-5}$ & $5.66 * 10^{-5}$ & $5.66 * 10^{-5}$ & $5.65^{*} 10^{-5}$ \\
Zhang [13], the 4th order differences & $1.13 * 10^{-6}$ & $3.22 * 10^{-6}$ & $3.22 * 10^{-6}$ & $3.22 * 10^{-6}$ & $3.22^{*} 10^{-6}$ \\
Uniform mesh using weak formulation & $4.80^{-6} 10^{-6}$ & $4.81 * 10^{-6}$ & $4.81 * 10^{-6}$ & $4.81 * 10^{-6}$ & $4.81 * 10^{-6}$ \\
\hline
\end{tabular}

Table 2 Maximal error comparison among different methods.

\begin{tabular}{|c|c|c|c|c|c|}
\hline \multirow{2}{*}{ Method } & & \multicolumn{4}{|c|}{ Maximal error } \\
\hline & & $\varepsilon=10^{-4}$ & $\varepsilon=10^{-6}$ & $\varepsilon=10^{-8}$ & $\varepsilon=10^{-10}$ \\
\hline Lin et al. [5] & 1,024 & $\begin{array}{l}9.26 * 10^{-6} \\
2.22 * 10^{-4}\end{array}$ & $\begin{array}{l}9.57 * 10^{-6} \\
3.97 * 10^{-5}\end{array}$ & $9.57 * 10^{-6}$ & $9.57 * 10^{-6}$ \\
\hline Zhang [2] & $N_{b}=600$ & $\begin{array}{l}5.17 * 10^{-5} \\
2.22 * 10^{-4}\end{array}$ & $\begin{array}{l}3.76 * 10^{-5} \\
2.22 * 10^{-4}\end{array}$ & $3.74 * 10^{-5}$ & $3.76^{*} 10^{-5}$ \\
\hline Uniform Mesh using Weak Form & $N=400$ & $4.33 * 10^{-5}$ & $\begin{array}{l}3.73 * 10^{-5} \\
2.22 * 10^{-4}\end{array}$ & $3.72 * 10^{-5}$ & $3.73 * 10^{-5}$ \\
\hline
\end{tabular}

$N_{b}$ is the number of points on the boundary layer.

Table 3 Maximal error comparison among different methods with $\varepsilon=10^{-10}$.

\begin{tabular}{lll}
\hline Method & Number of points & Max error \\
\hline Standard Shishkin mesh, Linss et al. [3] & 1,024 & $1.21 * 10^{-1}$ \\
Bakhvalov-Shishkinmesh, Linss et al. [3] & 1,024 & $2.63 * 10^{-3}$ \\
Nonuniform mesh, Lin et al. [5] & 400 (Non layer 100 + Layer 300) & $3.82 * 10^{-5}$ \\
Uniform mesh, Zhang [2] & 400 & $3.43 * 10^{-5}$ \\
Uniform mesh using weak formulation & 400 & $3.73 * 10^{-5}$ \\
\hline
\end{tabular}

also listed the maximal errors with different values of the singular perturbation parameter (Table 2). As expected and shown from the table, the new method is convergent and stable. Moreover, we used less number of mesh points for different levels of the singular perturbation and the numerical error is controlled at the same level.

We also compare the new method, a uniform mesh with weak formulation, with the methods using Shishkin type meshes from Linss et al. [3], in Table 3. Our numerical results are better with less number of mesh points. We achieved the error at $10^{-5}$ level with 400 mesh points while the methods with Shishkin type meshes gave an error at $10^{-3}$ level with 1,024 points.

\section{References}

[1] Chang, K. W., and Howes, F. A. 1984. Nonlinear Singular Perturbation Phenomena: Theory and Application. New York: Spring-Verlag.
[2] Zhang, W. 2006. "Numerical Solutions of Linear and Nonlinear Singular Perturbation Problems." PhD dissertation, University of Wisconsin Milwaukee.

[3] Linss, T., Roos, H., and Vulanovic, R. 2000. "Uniform Pointwise Convergence on Shishkin Type Meshes for Quasi-Linear Convection-Diffusion Problems." SIAM J.NUMER. ANAL. 38 (3): 897-912.

[4] Miller, J. J. H., O'Riordan, E., and Shishkin, G. I. 1996. Fitted Numerical Methods for Singular Perturbation Problems. Singapore: World Scientific Publishing Co.

[5] Lin, T. C., Schultz, D. H., and Zhang, W. 2008. "Numerical Solutions of Linear and Nonlinear Singular Perturbation Problems." Computers \& Math. Applic. 55 (11): 2574-92.

[6] Choudhury, S. R. 1996. "Nonstandard Difference Schemes of Nonlinear Singular Perturbation Problems." Int. J. of Applied Sc. \& Computations 2: 375-92.

[7] Ilicasuand, F. O., and Schultz, D. H. 2002. "High Order Methods for Singular Perturbation Problems." Computers Math. Applic. 47: 391-417.

[8] Segal, A. 1982. "Aspects of Numerical Methods for Elliptic Singular Perturbation Problems." SIAM J.SCI.COMPUT. 3 (3): 327-49.

[9] Keller, H. B. 1968. Numerical Methods for Two-Point 
Perturbed Differential Equations

Boundary Value Problems. Waltham, MA: Blaisdell Publishing Company.

[10] Lax, P. D., and Milgram, A. N. 1954. "Contributions to the Theory of Partial Differential Equations." Annals of Mathematics Studies 33: 167-90.

[11] El-Mistikawy, T. M., and Werle, M. J. 1978. "Numerical Method of Boundary Layers with Blowing-The
Exponential Box Scheme." AIAA J.: 749-51.

[12] O’Malley Jr., R. E. 1974. Introduction to Singular Perturbations. New York: Academic Press.

[13] Choo, J. Y., and Schultz, D. H. 1993. "High Order Methods for Differential Equations with Small Coefficients for the Second Order Terms." Computers Math. Applic. 25 (1): 105-23. 